\title{
Author Index Volume 27 (2011)
}

The issue number is given in front of the pagination

Aarsland, D., see Grambaite, R. (2) 453-462

Abd-El-Aziz, A.S., see Hashioka, S. (3) 531-541

Abdo, W.F., see Aerts, M.B. (2) 377-384

Adame, A., see Rockenstein, E. (4) 743-752

Aerts, M.B., R.A.J. Esselink, J.A.H.R. Claassen, W.F. Abdo, B.R. Bloem and M.M. Verbeek, CSF Tau, $\mathrm{A} \beta_{42}$, and MHPG Differentiate Dementia with Lewy Bodies from Alzheimer's Disease (2) 377384

Albertini, V., see Carecchio, M. (4) 781-790

Alikhani, N., L. Guo, S. Yan, H. Du, C.M. Pinho, J. Xi Chen, E. Glaser and S.S. Yan, Decreased Proteolytic Activity of the Mitochondrial Amyloid- $\beta$ Degrading Enzyme, PreP Peptidasome, in Alzheimer's Disease Brain Mitochondria (1) 75-87

Almasy, L., see Greenwood, T.A. (4) 897-907

Alonso, M.-D., see Lloret, A. (4) 701-709

Amarnath, V., see Davies, S.S. (1) 49-59

Ames, D., see Faux, N.G. (4) 909-922

Amieva, H., see Pérès, K. (1) 39-47

Amiry-Moghaddam, M., see Yang, J. (4) 711-722

Andreasen, M., see Du, X.-t. (2) 401-413

Andreasson, U., see Bjerke, M. (3) 665-676

Andrei, A., see de Toledo Ferraz Alves, T.C. (3) 575589

Angelo, G., see Greenwood, T.A. (4) 897-907

Arab, L., M.L. Biggs, E.S. O’Meara, W.T. Longstreth, P.K. Crane and A.L. Fitzpatrick, Gender Differences in Tea, Coffee, and Cognitive Decline in the Elderly: The Cardiovascular Health Study (3) 553-566

Archetti, S., see Picco, A. (4) 737-742

Arlt, S., see Jessen, F. (2) 393-399

Arnaldi, D., see Picco, A. (4) 737-742

Ashford, J.W., E. Gere and P.J. Bayley, Measuring Memory in Large Group Settings Using a Continuous Recognition Test (4) 885-895

Atwood, C.S., see Clark, I.A. (4) 691-699

Auriacombe, S., see Pérès, K. (1) 39-47

Badia, M.-C., see Lloret, A. (4) 701-709

Bandaru, V.V.R., see Mielke, M.M. (2) 259-269
Banerjee, K., see Sinha, M. (4) 753-765

Barbeau, E.J., see Didic, M. (1) 11-22

Barberger-Gateaua, P., see Pérès, K. (1) 39-47

Barcikowska, M., see Maruszak, A. (1) 197-210

Baron, R., see Fisher, Y. (1) 99-111

Bastos, O., see Correia, C.C. (1) 177-185

Basu, S., see Sinha, M. (4) 753-765

Bauer, C., R. Pardossi-Piquard, J. Dunys, M. Roy and F. Checler, $\gamma$-Secretase-Mediated Regulation of Neprilysin: Influence of Cell Density and Aging on Modulation by Imatinib (3) 511-520

Baum, E., see Reese, J.P. (4) 723-736

Bayley, P.J., see Ashford, J.W. (4) 885-895

Beach, T.G., see Hunter, J.M. (2) 361-376

Beal, M.F., see Dumont M. (1) 211-223

Bedford, L., see Williamson, R. (3) 615-625

Beeri, M.S., see Greenwood, T.A. (4) 897-907

Behera, P., see Sinha, M. (4) 753-765

Belrose, J., see Yang, K. (2) 243-252

Benisty, S., see Dumurgier, J. (4) 845-851

Benussi, L., see Carecchio, M. (4) 781-790

Benvenuti, S., see Cecchi, C. (3) 651-664

Berciano, J., see Vázquez-Higuera, J.L. (2) 291-297

Berg, D., see Maetzler, W. (1) 119-126

Bernoud-Hubac, N., see Davies, S.S. (1) 49-59

Bhaskerrao, S., see Britt, W.G. (3) 543-551

Bhowmick, P., see Sinha, M. (4) 753-765

Biggs, M.L., see Arab, L. (3) 553-566

Bihaqi, S.W., H. Huang, J. Wu and N.H. Zawia, Infant Exposure to Lead $(\mathrm{Pb})$ and Epigenetic Modifications in the Aging Primate Brain: Implications for Alzheimer's Disease (4) 819833

Binetti, G., see Carecchio, M. (4) 781-790

Biocca, S., see Canu, N. (3) 603-613

Bjerke, M., H. Zetterberg, Å. Edman, K. Blennow, A. Wallin and U. Andreasson, Cerebrospinal Fluid Matrix Metalloproteinases and Tissue Inhibitor of Metalloproteinases in Combination with Subcortical and Cortical Biomarkers in Vascular Dementia and Alzheimer's Disease (3) 665-676

Blanchard, J., see Rockenstein, E. (4) 743-752

Blennow, K., see Bjerke, M. (3) 665-676 
Block, W., see Jessen, F. (2) 393-399

Bloem, B.R., see Aerts, M.B. (2) 377-384

Bodine, C., see Davies, S.S. (1) 49-59

Borghi, R., see Guglielmotto, M. (4) 871-883

Borroni, B., see Carecchio, M. (4) 781-790

Bowers, W.J., see Hunter, J.M. (2) 361-376

Branicki, W., see Maruszak, A. (1) 197-210

Bresolin, N., see Carecchio, M. (4) 781-790

Britt, W.G., A.M. Hansen, S. Bhaskerrao, J.P. Larsen, F. Petersen, A. Dickson, C. Dickson and W.M. Kirsch, Mild Cognitive Impairment: Prodromal Alzheimer's Disease or Something Else? (3) 543551

Brodtmann, A., see Darby, D.G. (3) 627-637

Broersena, L.M., see de Wilde, M.C. (2) 327-339

Bullido, M.J., see Vázquez-Higuera, J.L. (2) 291-297

Busatto, G.F., see de Toledo Ferraz Alves, T.C. (3) 575589

Bush, A.I., see Faux, N.G. (4) 909-922

Caballero, L., see Cornejo, A. (1) 143-153

Calero, M., see Vázquez-Higuera, J.L. (2) 291-297

Calingasan, N.Y., see Dumont M. (1) 211-223

Calon, F., see Lebbadi, M. (4) 853-869

Camacho, K., see Greenwood, T.A. (4) 897-907

Camassa, L.M.A., see Yang, J. (4) 711-722

Campistol, J., see Ortez, C. (2) 253-257

Campos, M.S., see Correia, C.C. (1) 177-185

Canistro, D., see Puoliväli, J. (3) 499-510

Canter, J.A., see Maruszak, A. (1) 197-210

Cantoni, C., see Carecchio, M. (4) 781-790

Canu, N., I. Filesi, A. Pristerà, M.T. Ciotti and S. Biocca, Altered Intracellular Distribution of $\operatorname{PrP}^{\mathrm{C}}$ and Impairment of Proteasome Activity in Tau Overexpressing Cortical Neurons (3) 603-613

Cappa, S.F., see Cerami, C. (4) 791-797

Carbone, I., see Licastro, F. (4) 809-817

Carcaillon, L., see Pérès, K. (1) 39-47

Cardoso, I., see Oliveira, S.M. (2) 429-439

Carecchio, M., C. Fenoglio, F. Cortini, C. Comi, L. Benussi, R. Ghidon, B. Borroni, M. De Riz, M. Serpente, C. Cantoni, M. Franceschi, V. Albertini, F. Monaco, I. Rainero, G. Binetti, A. Padovani, N. Bresolin, E. Scarpini and D. Galimberti, Cerebrospinal Fluid Biomarkers in Progranulin Mutations Carriers (4) 781-790

Carrión-Baralt, J.R., see Greenwood, T.A. (4) 897-907

Caruana, M., see Gauci, A.J. (4) 767-779

Casado, M., see Ortez, C. (2) 253-257

Cascella, R., see Cecchi, C. (3) 651-664

Ceccaldi, M., see Didic, M. (1) 11-22
Cecchi, C., E. Evangelisti, R. Cascella, M. Zampagni, S. Benvenuti, P. Luciani, C. Deledda, I. Cellai, D. Wright, R. Saccardi, A. Peri and M. Stefani, Neuronal Differentiation of Human Mesenchymal Stromal Cells Increases their Resistance to $\mathrm{A} \beta_{42}$ Aggregate Toxicity (3) 651-664

Cellai, I., see Cecchi, C. (3) 651-664

Cerami, C., A. Marcone, D. Galimberti, C. Villa, E. Scarpini and S.F. Cappa, From Genotype to Phenotype: Two Cases of Genetic Frontotemporal Lobar Degeneration with Premorbid Bipolar Disorder (4) 791-797

Chabriat, H., see Dumurgier, J. (4) 845-851

Chakrabarti, S., see Sinha, M. (4) 753-765

Chasseigneaux, S., see Dumurgier, J. (4) 845-851

Checler F., see Bauer, C. (3) 511-520

Chen, M., H.T. Nguyen and D.R. Sawmiller, What to Look for Beyond "Pathogenic" Factors in Senile Dementia? A Functional Deficiency of $\mathrm{Ca}^{2+}$ Signaling (4) 679-689

Choi, D.-Y., see Choi, I.S. (1) 127-141

Choi, I.S., Y.-J. Lee, D.-Y. Choi, Y.K. Lee, Y.H. Lee, K.H. Kim, Y.H. Kim, Y.H. Jeon, E.H. Kim, S.B. Han, J.K. Jung, Y.P. Yun, K.-W. Oh, D.Y. Hwang and J.T. Hong, 4-O-Methylhonokiol Attenuated Memory Impairment Through Modulation of Oxidative Damage of Enzymes Involving Amyloid- $\beta$ Generation and Accumulation in a Mouse Model of Alzheimer's Disease (1) 127-141

Ciotti, M.T., see Canu, N. (3) 603-613

Cirrito, J.R., see Kou, J. (1) 23-38

Claassen, J.A.H.R., see Aerts, M.B. (2) 377-384

Clark I.A. and C.S. Atwood, Is TNF a Link between Aging-Related Reproductive Endocrine Dyscrasia and Alzheimer's Disease? (4) 691-699

Cole, A.R., see Williamson, R. (3) 615-625

Combarros, O., see Vázquez-Higuera, J.L. (2) 291-297

Comi, C., see Carecchio, M. (4) 781-790

Coons, S.W., see Yin, J.-X. (1) 89-98

Cornejo, A., J.M. Jiménez, L. Caballero, F. Melo and R.B. Maccioni, Fulvic Acid Inhibits Aggregation and Promotes Disassembly of Tau Fibrils Associated with Alzheimer's Disease (1) 143-153

Correia, C.C., F. Lima, F. Junqueira, M.S. Campos, O. Bastos, K. Petribú, J. Laks and J.E. Galvin, AD8-Brazil: Cross-Cultural Validation of the Ascertaining Dementia Interview in Portuguese (1) 177-185

Cortini, F., see Carecchio, M. (4) 781-790

Crane, P.K., see Arab, L. (3) 553-566

Crews, L., see Rockenstein, E. (4) 743-752 
D’Adamio, L., see Matsuda, S. (2) 385-391

Dannhoff, F., see Reese, J.P. (4) 723-736

Darby, D.G., A. Brodtmann, R.H. Pietrzak, J. Fredrickson, M. Woodward, V.L. Villemagne, A. Fredrickson, P. Maruff and C. Rowe, Episodic Memory Decline Predicts Cortical Amyloid Status in Community-Dwelling Older Adults (3) 627637

Darby, E., see Mielke, M.M. (2) 259-269

Dartigues, J.-F., see Pérès, K. (1) 39-47

Daugs, I.D., see Hunter, J.M. (2) 361-376

David, R., L. Friedman, E. Mulin, A. Noda, F. Le Duff, Q. Kennedy, R. Garcia, P.H. Robert, J.A. Yesavage, J.M. Zeitzer, Lack of Association Between COMT Polymorphisms and Apathy in Alzheimer's Disease (1) 155-161

Davies, K.J.A., see Lloret, A. (4) 701-709

Davies, S.S., C. Bodine, E. Matafonova, B.G. Pantazides, N. Bernoud-Hubac, F.E. Harrison, S.J. Olson, T.J. Montine, V. Amarnath and L. Jackson Roberts, Treatment with a $\gamma$-Ketoaldehyde Scavenger Prevents Working Memory Deficits in hApoE4 Mice (1) 49-59

de Leon, M., see Mosconi, L. (3) 483-490

De Riz, M., see Carecchio, M. (4) 781-790

de Souza Duran, F.L., see de Toledo Ferraz Alves, T.C. (3) 575-589

de Toledo Ferraz Alves, T.C., M. Scazufca, P. Squarzoni, F.L. de Souza Duran, J.H. Tamashiro-Duran, H.P. Vallada, A. Andrei, M. Wajngarten, P.R. Menezes and G.F. Busatto, Subtle Gray Matter Changes in Temporo-Parietal Cortex Associated with Cardiovascular Risk Factors (3) 575-589

de Wilde, M.C., B. Penke, E.M. van der Beek, A.A.M. Kuipers, P.J. Kamphuis and L.M. Broersena, Neuroprotective Effects of a Specific MultiNutrient Intervention Against $\mathrm{A} \beta_{42}$-Induced Toxicity in Rats (2) 327-339

Deledda, C., see Cecchi, C. (3) 651-664

Devic, I., see Shi, M. (2) 299-305

di Lorenzo, D., see Picco, A. (4) 737-742

Dickson, A., see Britt, W.G. (3) 543-551

Dickson, C., see Britt, W.G. (3) 543-551

Didic, M., E.J. Barbeau, O. Felician, E. Tramoni, E. Guedj, M. Poncet and M. Ceccaldi, Which Memory System is Impaired First in Alzheimer's Disease? (1) 11-22

Dobato, J.L., see Vázquez-Higuera, J.L. (2) 291-297

Dodel, R., see Reese, J.P. (4) 723-736

Doody, R.S., see Mielke, M.M. (2) 259-269

Doppler, E., see Rockenstein, E. (4) 743-752
Du, H., see Alikhani, N. (1) 75-87

Du, H., see Li, C. (2) 281-290

Du, X.-t., L. Wang, Y.-j. Wang, M. Andreasen, D.-w. Zhan, Y. Feng, M. Li, M. Zhao, D. Otzen, D. Xue, Y. Yang and R.-t. Liu, $A \beta_{1-16}$ Can Aggregate and Induce the Production of Reactive Oxygen Species, Nitric Oxide, and Inflammatory Cytokines (2) 401-413

Duarte, S.T., see Ortez, C. (2) 253-257

Dumont, M., K. Kipiani, F. Yu, E. Wille, M. Katz, N.Y. Calingasan, G.K. Gouras, M.T. Lin and M.F. Beal Coenzyme Q10 Decreases Amyloid Pathology and Improves Behavior in a Transgenic Mouse Model of Alzheimer's Disease (1) 211-223

Dumurgier, J., C. Paquet, K. Peoc'h, P. Lapalus, F. Mouton-Liger, S. Benisty, S. Chasseigneaux, H. Chabriat and J. Hugon, CSF A $\beta_{1-42}$ Levels and Glucose Metabolism in Alzheimer's Disease (4) 845-851

Dunys, J., see Bauer, C. (3) 511-520

Edgar, J.S., see Shi, M. (2) 299-305

Edman, A., see Bjerke, M. (3) 665-676

Ellis, K.A., see Faux, N.G. (4) 909-922

Emond, V., see Lebbadi, M. (4) 853-869

Ende, G., see Jessen, F. (2) 393-399

Erkinjuntti, T., see Verdelho, A. (3) 491-498

Ermak, G., see Lloret, A. (4) 701-709

Esselink, R.A.J., see Aerts, M.B. (2) 377-384

Etschmaier, K., see Schweinzer, C. (2) 341-360

Evangelisti, E., see Cecchi, C. (3) 651-664

Evin, G., see Kim, W.S. (2) 441-452

Faux, N.G., K.A. Ellis, L. Porter, C.J. Fowler, S.M. Laws, R.N. Martins, K.K. Pertile, A. Rembach, C.C. Rowe, R.L. Rumble, C. Szoeke, K. Taddei, T. Taddei, B.O. Trounson, V.L. Villemagne, V. Ward, D. Ames, C.L. Masters and A.I. Bush, Homocysteine, Vitamin B12, and Folic Acid Levels in Alzheimer's Disease, Mild Cognitive Impairment, and Healthy Elderly: Baseline Characteristics in Subjects of the Australian Imaging Biomarker Lifestyle Study (4) 909-922

Fazekas, F., see Verdelho, A. (3) 491-498

Felician, O., see Didic, M. (1) 11-22

Feng, Y., see Du, X.-t. (2) 401-413

Fenoglio, C., see Carecchio, M. (4) 781-790

Ferrara, M., see Picco, A. (4) 737-742

Ferro, J.M., see Verdelho, A. (3) 491-498

Filesi, I., see Canu, N. (3) 603-613 
Fisher, Y., A. Nemirovsky, R. Baron and A. Monsonego, Dendritic Cells Regulate Amyloid-B-Specific T-Cell Entry into the Brain: The Role of Perivascular Amyloid-B (1) 99-111

Fitzgerald, M.L., see Kim, W.S. (2) 441-452

Fitzpatrick, A.L., see Arab, L. (3) 553-566

Fladby, T., see Grambaite, R. (2) 453-462

Fons, C., see Ortez, C. (2) 253-257

Fornaro, M., see Guglielmotto, M. (4) 871-883

Fowler, C.J., see Faux, N.G. (4) 909-922

Franceschi, M., see Carecchio, M. (4) 781-790

Frank-García, A., see Vázquez-Higuera, J.L. (2) 291297

Fredrickson, A., see Darby, D.G. (3) 627-637

Fredrickson, J., see Darby, D.G. (3) 627-637

Freeman, M.W., see Kim, W.S. (2) 441-452

Friedman, L., see David, R., (1) 155-161

Frölich, L., see Jessen, F. (2) 393-399

Fukuchi, K.-i., see Kou, J. (1) 23-38

Furay, A.R., see Shi, M. (2) 299-305

Gabryelewicz, T., see Maruszak, A. (1) 197-210

Galimberti, D., see Carecchio, M. (4) 781-790

Galimberti, D., see Cerami, C. (4) 791-797

Galvin, J.E., see Correia, C.C. (1) 177-185

Gao, B., Z. Long, L. Zhao and G. He, Effect of Normobaric Hyperoxia on Behavioral Deficits and Neuropathology in Alzheimer's Disease Mouse Model (2) 317-326

Garcia, R., see David, R., (1) 155-161

García-Cazorla, A., see Ortez, C. (2)253-257

García-Villoria, J., see Ortez, C. (2)253-257

Garner, B., see Kim, W.S. (2) 441-452

Gauci, A.J., M. Caruana, A. Giese, C. Scerri and N. Vassallo, Identification of Polyphenolic Compounds and Black Tea Extract as Potent Inhibitors of Lipid Membrane Destabilization by A $\beta_{42}$ Aggregates (4) 767-779

Gawęda-Walerych, K., see Maruszak, A. (1) 197-210

Geider, J., see Seidl, U. (3) 567-574

Geldsetzer, M.-B., see Reese, J.P. (4) 723-736

Gere, E., see Ashford, J.W. (4) 885-895

Ghidon, R., see Carecchio, M. (4) 781-790

Giese, A., see Gauci, A.J. (4) 767-779

Giliberto, L., see Guglielmotto, M. (4) 871-883

Ginghina, C., see Shi, M. (2) 299-305

Giraldo, E., see Lloret, A. (4) 701-709

Gjerstad, L., see Grambaite, R. (2) 453-462

Glaser, E., see Alikhani, N. (1) 75-87

Gold, B.T., see Threlkeld, Z.D. (2) 415-427

Gonzalez-Cuyar, L.F., see Shi, M. (2) 299-305
Goodlett, D.R., see Shi, M. (2) 299-305

Gouras, G.K., see Dumont M. (1) 211-223

Grambaite, R., P. Selnes, I. Reinvang, D. Aarsland, E. Hessen, L. Gjerstad and T. Fladby, Executive Dysfunction in Mild Cognitive Impairment is Associated with Changes in Frontal and Cingulate White Matter Tracts (2) 453-462

Gray, A.M., Luengo-Fernandez, R. (1) 187-196

Greenwood, T.A., M.S. Beeri, J. Schmeidler, D. Valerio, H. Raventós, L. Mora-Villalobos, K. Camacho, J.R. Carrión-Baralt, G. Angelo, L. Almasy, M. Sano and J.M. Silverman, Heritability of Cognitive Functions in Families of Successful Cognitive Aging Probands from the Central Valley of Costa Rica (4) 897-907

Grundke-Iqbal, I., see Rockenstein, E. (4) 743-752

Guan, J.-W., C.-Q. Huang, Y.-H. Li, C.-M. Wan, C. You, Z.-R. Wang, Y.-Y. Liu and Q.-X. Liu, No Association Between Hypertension and Risk for Alzheimer's Disease: A Meta-Analysis of Longitudinal Studies (4) 799-807

Guedj, E., see Didic, M. (1) 11-22

Guglielmotto, M., D. Monteleone, L. Giliberto, M. Fornaro, R. Borghi, E. Tamagno and M. Tabaton, Amyloid- $\beta_{42}$ Activates the Expression of BACE1 Through the JNK Pathway (4) 871-883

Gui, L., see Li, C. (2) 281-290

Guo, L., see Alikhani, N. (1) 75-87

Gür, O., see Jessen, F. (2) 393-399

Gusev, N.B., see Sluchanko, N.N. (3) 467-476

Hammen, T., see Jessen, F. (2) 393-399

Han, S.B., see Choi, I.S. (1) 127-141

Hansen, A.M., see Britt, W.G. (3) 543-551

Harrison, F.E., see Davies, S.S. (1) 49-59

Hashioka, S., J.G. McLarnon, J.K. Ryu, A.M. Youssef, A.S. Abd-El-Aziz, E.G. Neeland and A. Klegeris, Pyrazole Compound 2-MBAPA as a Novel Inhibitor of Microglial Activation and Neurotoxicity in vitro and in vivo (3) 531-541

Haughey, N.J., see Mielke, M.M. (2) 259-269

Hauser, A.-K., see Maetzler, W. (1) 119-126

He, G., see Gao, B. (2) 317-326

Heikkinen, T., see Puoliväli, J. (3) 499-510

Helmer, C., see Pérès, K. (1) 39-47

Henkel, D., see Reese, J.P. (4) 723-736

Hessen, E., see Grambaite, R. (2) 453-462

Heßmann, P., see Reese, J.P. (4) 723-736

Heyny-von Haußen, R., see Taghavi, A. (4) 835-843

Hill, A.F., see Kim, W.S. (2) 441-452

Himeno, E., see Ma, L. (1) 225-237 
Hirohata, M., see Takasaki, J. (2) 271-280

Hirzmann, P., see Reese, J.P. (4) 723-736

Hong, J.T., see Choi, I.S. (1) 127-141

Howlett, D., see Williamson, R. (3) 615-625

Huang, C.-Q., see Guan, J.-W. (4) 799-807

Huang, H., see Bihaqi, S.W. (4) 819-833

Hugon, J., see Dumurgier, J. (4) 845-851

Hunter, J.M., W.J. Bowers, C.L. Maarouf, M.A. Mastrangelo, I.D. Daugs, T.A. Kokjohn, W.M. Kalbacka, D.C. Luehrs, J. Valla, T.G. Beach and A.E. Roher, Biochemical and Morphological Characterization of the AßPP/PS/Tau Triple Transgenic Mouse Model and its Relevance to Sporadic Alzheimer's Disease (2) 361-376

Hwang, D.Y., see Choi, I.S. (1) 127-141

Hwang, H., see Shi, M. (2) 299-305

Ianni, M., see Licastro, F. (4) 809-817

Iinuma, K.M., see Ma, L. (1) 225-237

Ikeda, T., see Takasaki, J. (2) 271-28

Inzitari, D., see Verdelho, A. (3) 491-498

Iqbal, K., see Rockenstein, E. (4) 743-752

Jackson Roberts, L., see Davies, S.S. (1) 49-59

Jackson, M.F., see Yang, K. (2) 243-252

Jacqmin-Gadda, H., see Pérès, K. (1) 39-47

Javier, E., see Mosconi, L. (3) 483-490

Jeon, E.H., see Choi, I.S. (1) 127-141

Jessen, F., P. Lewczuk, O. Gür, W. Block, G. Ende, L. Frölich, T. Hammen, S. Arlt, J. Kornhuber, T. Kucinski, J. Popp, O. Peters, W. Maier, F. Träber and J. Wiltfang, Association of N-Acetylaspartate and Fluid $A \beta_{42}$ in Dementia (2) 393-399

Jessen, F., see Reese, J.P. (4) 723-736

Jicha, G.A., see Threlkeld, Z.D. (2) 415-427

Jiménez, J.M., see Cornejo, A. (1) 143-153

Julien, C., see Lebbadi, M. (4) 853-869

Jung, J.K., see Choi, I.S. (1) 127-141

Junqueira, F., see Correia, C.C. (1) 177-185

Kalbacka, W.M., see Hunter, J.M. (2) 361-376

Kalesnykas, G., see Puoliväli, J. (3) 499-510

Kamphuis, P.J., see de Wilde, M.C. (2) 327-339

Kang, J.X., see Lebbadi, M. (4) 853-869

Katz, M., see Dumont, M. (1) 211-223

Kavanagh, S., B. Van Baelen and B. Schäuble, LongTerm Effects of Galantamine on Cognitive Function in Alzheimer's Disease: A Large-Scale International Retrospective Study (3) 521-530

Kennedy, Q., see David, R. (1) 155-161

Kim, E.H., see Choi, I.S. (1) 127-141
Kim, H., see Kou, J. (1) 23-38

Kim, K.H., see Choi, I.S. (1) 127-141

Kim, W.S., A.F. Hill, M.L. Fitzgerald, M.W. Freeman, G. Evin and B. Garner, Wild Type and Tangier Disease ABCA1 Mutants Modulate Cellular Amyloid- $\beta$ Production Independent of Cholesterol Efflux Activity (2) 441-452

Kim, Y.H., see Choi, I.S. (1) 127-141

Kipiani, K., see Dumont, M. (1) 211-223

Kira, J.-i., see Ma, L. (1) 225-237

Kirsch, W.M., see Britt, W.G. (3) 543-551

Klegeris, A., see Hashioka, S. (3) 531-541

Kober, A., see Schweinzer, C. (2) 341-360

Koikkalainen, J., see Mattila, J. (1) 163-176

Kokjohn, T.A., see Hunter, J.M. (2) 361-376

Kornhuber, J., see Jessen, F. (2) 393-399

Kou, J., H. Kim, A. Pattanayak, M. Song, J.-E. Lim, H. Taguchi, S. Paul, J.R. Cirrito, S. Ponnazhagan and K.-i. Fukuchi, Anti-Amyloid-B Single-Chain Antibody Brain Delivery Via AAV Reduces Amyloid Load But May Increase Cerebral Hemorrhages in an Alzheimer's Disease Mouse Model (1) 23-38

Koutras, C., C.B. Lessard and G. Lévesque, A Nuclear Function for the Presenilin 1 Neuronal Partner NPRAP/ $\delta$-Catenin (2) 307-316

Kresse, A., see Schweinzer, C., (2) 341-360

Kucinski, T., see Jessen, F. (2) 393-399

Kuipers, A.A.M., see de Wilde, M.C. (2) 327-339

Laks, J., see Correia, C.C. (1) 177-185

Lang, I., see Schweinzer, C., (2) 341-360

Lannfelt, L., see Yang, J. (4) 711-722

Lapalus, P., see Dumurgier, J. (4) 845-851

Larsen, J.P., see Britt, W.G. (3) 543-551

Laws, S.M., see Faux, N.G. (4) 909-922

Le Duff, F., see David, R. (1) 155-161

Leal, J., see Luengo-Fernandez, R. (1) 187-196

Lebbadi, M., C. Julien, A. Phivilay, C. Tremblay, V. Emond, J.X. Kang and F. Calon, Endogenous Conversion of Omega-6 into Omega-3 Fatty Acids Improves Neuropathology in an Animal Model of Alzheimer's Disease (4) 853-869

Lee, Y.H., see Choi, I.S. (1) 127-141

Lee, Y.-J., see Choi, I.S. (1) 127-141

Lee, Y.K., see Choi, I.S. (1) 127-141

Lehtimäki, K., see Puoliväli, J. (3) 499-510

Lei, G., see Yang, K. (2) 243-252

Lerche, S., see Maetzler, W. (1) 119-126

Lessard, C.B., see Koutras, C. (2) 307-316 
Leszek, J., see Zimny, A. (3) 591-601

Lévesque, G., see Koutras, C. (2) 307-316

Lewczuk, P., see Jessen, F. (2) 393-399

Lezi, E., see Mosconi, L. (3) 483-490

Li, B., see Rockenstein, E. (4) 743-752

Li, C., J. Wang, L. Gui, J. Zheng, C. Liu and H. Du, Alterations of Whole-Brain Cortical Area and Thickness in Mild Cognitive Impairment and Alzheimer's Disease (2) 281-290

Li, G., see Shi, M. (2) 299-305

Li, M., see Du, X.-t. (2) 401-413

Licastro, F., I. Carbone, M. Ianni and E. Porcellini, Gene Signature in Alzheimer's Disease and Environmental Factors: The Virus Chronicle (4) 809-817

Li, Y.-H., see Guan, J.-W. (4) 799-807

Lim, J.-E., see Kou, J. (1) 23-38

Lima, F., see Correia, C.C. (1) 177-185

Lin, H.-j., see Yin, J.-x. (1) 89-98

Lin, M.T., see Dumont M. (1) 211-223

Liu, C., see Li, C. (2) 281-290

Liu, G.-P., see Wei, W. (3) 639-650

Liu, Q.-X., see Guan, J.-W. (4) 799-807

Liu, R.-t., see Du, X.-t. (2) 401-413

Liu, Y.-H., see Wei, W. (3) 639-650

Liu, Y.-Y., see Guan, J.-W. (4) 799-807

Lloret, A., M.-C. Badia, E. Giraldo, G. Ermak, M.-D. Alonso, F.V. Pallardó, K.J.A. Davies and J. Viña, Amyloid- $\beta$ Toxicity and Tau Hyperphosphorylation are Linked Via RCAN1 in Alzheimer's Disease (4) 701-709

Lloyd, D.G., see Pac-Soo, C. (1) 1-10

Long, Z., see Gao, B. (2) 317-326

Longstreth, W.T., see Arab, L. (3) 553-566

Lötjönen, J., see Mattila, J. (1) 163-176

Lu, J., see Mosconi, L. (3) 483-490

Luca, R.M., see Saab, B.J. (3) 477-482

Luciani, P., see Cecchi, C. (3) 651-664

Luehrs, D.C., see Hunter, J.M. (2) 361-376

Lueken, U., see Seidl, U. (3) 567-574

Luengo-Fernandez, R., J. Leal and A.M. Gray, Cost of Dementia in the Pre-Enlargement Countries of the European Union (1) 187-196

Lunde, L.K., see Yang, J. (4) 711-722

Lyketsos, C.G., see Mielke, M.M. (2) 259-269

Lyutvinskiy, Y., see Yang, H. (1) 113-118

Ma, D., see Pac-Soo, C. (1) 1-10

Ma, L., Y. Ohyagi, N. Nakamura, K.M. Iinuma, K. Miyoshi, E. Himeno, N. Soejima, Y.T. Yanagihara,
N. Sakae, R. Yamasaki and J.-i. Kira, Activation of Glutathione Peroxidase and Inhibition of p53Related Apoptosis by Apomorphine (1) 225-237

Maarouf, C.L., see Hunter, J.M. (2) 361-376

Maccioni, R.B., see Cornejo, A. (1) 143-153

MacDonald, J.F., see Yang, K. (2) 243-252

Madureira, S., see Verdelho, A. (3) 491-498

Maetzler, W., A.K. Stapf, C. Schulte, A.-K. Hauser, S. Lerche, I. Wurster, E. Schleicher, A. Melms and D. Berg, Serum and Cerebrospinal Fluid Uric Acid Levels in Lewy Body Disorders: Associations with Disease Occurrence and Amyloid-ß Pathway (1) $119-126$

Maier, W., see Jessen, F. (2) 393-399

Mall, G., see Taghavi, A. (4) 835-843

Mandelkow, E., see Taghavi, A. (4) 835-843

Mandelkow, E.-M., see Taghavi, A. (4) 835-843

Mann, D.M.A., see Williamson, R. (3) 615-625

Mante, M., see Rockenstein, E. (4) 743-752

Marcone, A., see Cerami, C. (4) 791-797

Martins, R.N., see Faux, N.G. (4) 909-922

Maruff P., see Darby, D.G. (3) 627-637

Maruszak, A., K. Safranow, W. Branicki, K. GawędaWalerych, E. Pośpiech, T. Gabryelewicz, J.A. Canter, M. Barcikowska and C. Żekanowski, The Impact of Mitochondrial and Nuclear DNA Variants on Late-Onset Alzheimer's Disease Risk (1) $197-210$

Masliah, E., see Rockenstein, E. (4) 743-752

Masters, C.L., see Faux, N.G. (4) 909-922

Mastrangelo, M.A., see Hunter, J.M. (2) 361-376

Matafonova, E., see Davies, S.S. (1) 49-59

Mateo, I., see Vázquez-Higuera, J.L. (2) 291-297

Matharan, F., see Pérès, K. (1) 39-47

Matsuda, S., R. Tamayev and L. D'Adamio, Increased AßPP Processing in Familial Danish Dementia Patients (2) 385-391

Mattila, J., J. Koikkalainen, A. Virkki, A. Simonsen, M. van Gils, G. Waldemar, H. Soininen and J. Lötjönen, A Disease State Fingerprint for Evaluation of Alzheimer's Disease (1) 163-176

Mayer, J., see Williamson, R. (3) 615-625

McHugh, P., see Mosconi, L. (3) 483-490

McLarnon, J.G., see Hashioka, S. (3) 531-541

Melms, A., see Maetzler, W. (1) 119-126

Melo, F., see Cornejo, A. (1) 143-153

Menezes, P.R., see de Toledo Ferraz Alves, T.C. (3) 575-589

Mielke, M.M., N.J. Haughey, V.V.R. Bandaru, D.D. Weinberg, E. Darby, N. Zaidi, V. Pavlik, R.S. Doody and C.G. Lyketsos, Plasma Sphingomyelins 
are Associated with Cognitive Progression in Alzheimer's Disease (2) 259-269

Miettinen, T.-K., see Puoliväli, J. (3) 499-510

Miyoshi, K., see Ma, L. (1) 225-237

Moessler, H., see Rockenstein, E. (4) 743-752

Moleiro, C., see Verdelho, A. (3) 491-498

Monaco, F., see Carecchio, M. (4) 781-790

Monsonego, A., see Fisher, Y. (1) 99-111

Monteleone, D., see Guglielmotto, M. (4) 871-883

Montine, T.J., see Davies, S.S. (1) 49-59

Mora-Villalobos, L., see Greenwood, T.A. (4) 897-907

Morbelli, S., see Picco, A. (4) 737-742

Morinaga, A., see Takasaki, J. (2) 271-280

Mosconi, L., M. de Leon, J. Murray, E. Lezi, J. Lu, E. Javier, P. McHugh and R.H. Swerdlow, Reduced Mitochondria Cytochrome Oxidase Activity in Adult Children of Mothers with Alzheimer's Disease (3) 483-490

Mousseau, D.D., see Wei, W. (3) 639-650

Mouton-Liger, F., see Dumurgier, J. (4) 845-851

Mulin, E., see David, R., (1) 155-161

Müller, M.J., see Reese, J.P. (4) 723-736

Murray, J., see Mosconi, L. (3) 483-490

Nakamura, N., see Ma, L. (1) 225-237

Nasir, S., see Taghavi, A. (4) 835-843

Neeland, E.G., see Hashioka, S. (3) 531-541

Nemirovsky, A., see Fisher, Y. (1) 99-111

Nguyen, H.T., see Chen, M., (4) 679-689

Nilsson, L.N.G., see Yang, J. (4) 711-722

Nobili, F., see Picco, A. (4) 734-742

Noda, A., see David, R., (1) 155-161

Noga, L., see Zimny, A. (3) 591-601

Novak, P., see Rockenstein, E. (4) 743-752

Nuntagij, P., see Yang, J. (4) 711-722

Nurmi, A., see Puoliväli, J. (3) 499-510

O’Meara, E.S., see Arab, L. (3) 553-566

Oguchi, T., see Yang, J. (4) 711-722

Oh, K.-W., see Choi, I.S. (1) 127-141

Ohyagi, Y., see Ma, L. (1) 225-237

Oliveira, S.M., C.A. Ribeiro, I. Cardoso and M.J. Saraiva, Gender-Dependent Transthyretin Modulation of Brain Amyloid- $\$$ Levels: Evidence from a Mouse Model of Alzheimer's Disease (2) 429-439

Olson, S.J., see Davies, S.S. (1) 49-59

Ono, K., see Takasaki, J. (2) 271-280

Orgogozo, J.-M., see Pérès, K. (1) 39-47

Ormazábal, A., see Ortez, C. (2) 253-257

Ortez, C., C. Villar, C. Fons, S.T. Duarte, A. Pérez, J. García-Villoria, A. Ribes, A. Ormazábal, M.
Casado, J. Campistol, M.A. Vilaseca and A. GarcíaCazorla, Undetectable Levels of CSF Amyloid- $\beta$ Peptide in a Patient with $17 \beta$-Hydroxysteroid Dehydrogenase Deficiency (2) 253-257

Ottersen, O.P., see Yang, J. (4) 711-722

Otzen, D., see Du, X.-t. (2) 401-413

Pac-Soo, C., D.G. Lloyd, M.P. Vizcaychipi and D. Ma, Statins: The Role in the Treatment and Prevention of Alzheimer's Neurodegeneration (1) 1-10

Padovani, A., see Carecchio, M. (4) 781-790

Pallardó, F.V., see Lloret, A. (4) 701-709

Pan, C., see Shi, M. (2) 299-305

Pantazides, B.G., see Davies, S.S. (1) 49-59

Pantoni, L., see Verdelho, A. (3) 491-498

Panzenboeck, U., see Schweinzer, C. (2) 341-360

Paolini, M., see Puoliväli, J. (3) 499-510

Paquet, C., see Dumurgier, J. (4) 845-851

Pardossi-Piquard, R., see Bauer, C. (3) 511-520

Pattanayak, A., see Kou, J. (1) 23-38

Paul, S., see Kou, J. (1) 23-38

Pavlik, V., see Mielke, M.M. (2) 259-269

Penke, B., see de Wilde, M.C. (2) 327-339

Peoc'h, K., see Dumurgier, J. (4) 845-851

Pérès, K., C. Helmer, H. Amieva, F. Matharan, L. Carcaillon, H. Jacqmin-Gadda, S. Auriacombe, J.-M. Orgogozo, P. Barberger-Gateaua and J.-F. Dartigues, Gender Differences in the Prodromal Signs of Dementia: Memory Complaint and IADL-Restriction. A Prospective PopulationBased Cohort (1) 39-47

Pérez, A., see Ortez, C. (2) 253-257

Peri, A., see Cecchi, C. (3) 651-664

Pertile, K.K., see Faux, N.G. (4) 909-922

Peskind, E.R., see Shi, M. (2) 299-305

Peters, O., see Jessen, F. (2) 393-399

Petersen, F., see Britt, W.G. (3) 543-551

Petribú, K., see Correia, C.C. (1) 177-185

Phivilay, A., see Lebbadi, M. (4) 853-869

Piccini, A., see Picco, A. (4) 737-742

Picco, A., S. Archetti, M. Ferrara, D. Arnaldi, A. Piccini, C. Serrati, D. di Lorenzo, S. Morbelli and F. Nobili, Seizures Can Precede Cognitive Symptoms in Late-Onset Alzheimer's Disease (4) 737-742

Pickhardt, M., see Taghavi, A. (4) 835-843

Pietrzak, R.H., see Darby, D.G. (3) 627-637

Pinho, C.M., see Alikhani, N. (1) 75-87

Platt, B., see Williamson, R. (3) 615-625

Plattner, F., see Williamson, R. (3) 615-625

Poggesi, A., see Verdelho, A. (3) 491-498

Poncet, M., see Didic, M. (1) 11-22 
Ponnazhagan, S., see Kou, J. (1) 23-38

Popp, J., see Jessen, F. (2) 393-399

Porcellini, E., see Licastro, F. (4) 809-817

Porter, L., see Faux, N.G. (4) 909-922

Pośpiech, E., see Maruszak, A. (1) 197-210

Pozueta, A., see Vázquez-Higuera, J.L. (2) 291-297

Pristerà, A., see Canu, N. (3) 603-613

Puoliväli, J., A. Nurmi, T.-K. Miettinen, A. Soleti, F. Riccardino, G. Kalesnykas, T. Heikkinen, N. Vartiainen, R. Pussinen, L. Tähtivaara, K. Lehtimäki, J. Yrjänheikki, D. Canistro, A. Sapone, E. Spisni and M. Paolini, The Radical Scavenger IAC (bis(1-hydroxy- 2,2,6,6-tetramethyl-4piperidinyl) decantionate) Decreases Mortality, Enhances Cognitive Functions in Water Maze and Reduces Amyloid Plaque Burden in hAßPP Transgenic Mice (3) 499-510

Pussinen, R., see Puoliväli, J. (3) 499-510

Rainero, I., see Carecchio, M. (4) 781-790

Raventós, H., see Greenwood, T.A. (4) 897-907

Reese, J.P., P. Heßmann, G. Seeberg, D. Henkel, P. Hirzmann, J. Rieke, E. Baum, F. Dannhoff, M.J. Müller, F. Jessen, M.-B. Geldsetzer and R. Dodel, Cost and Care of Patients with Alzheimer's Disease: Clinical Predictors in German Health Care Settings (4) 723-736

Reinvang, I., see Grambaite, R. (2) 453-462

Rembach, A., see Faux, N.G. (4) 909-922

Ribeiro, C.A., see Oliveira, S.M. (2) 429-439

Ribes, A., see Ortez, C. (2) 253-257

Riccardino, F., see Puoliväli, J. (3) 499-510

Rieke, J., see Reese, J.P. (4) 723-736

Robert, P.H., see David, R. (1) 155-161

Rockenstein, E., K. Ubhi, E. Doppler, P. Novak, H. Moessler, B. Li, J. Blanchard, I. Grundke-Iqbal, K. Iqbal, M. Mante, A. Adame, L. Crews and E. Masliah, Regional Comparison of the Neurogenic Effects ofCNTF-DerivedPeptides and Cerebrolysin in A $\beta P P$ Transgenic Mice (4) 743-752

Roder, J.C., see Saab, B.J. (3) 477-482

Rodrigues, C.M.P., see Viana, R.J.S. (1) 61-73

Rodríguez-Rodríguez, E., see Vázquez-Higuera, J.L. (2) 291-297

Roher, A.E., see Hunter, J.M. (2) 361-376

Rowe, C., see Darby, D.G. (3) 627-637

Rowe, C.C., see Faux, N.G. (4) 909-922

Roy, M., see Bauer, C. (3) 511-520

Rumble, R.L., see Faux, N.G. (4) 909-922

Ryu, J.K., see Hashioka, S. (3) 531-541
Saab, A.M.P., see Saab, B.J. (3) 477-482

Saab, B.J., R.M. Luca, W.B. Yuen, A.M.P. Saab and J.C. Roder, Memantine Affects Cognitive Flexibility in the Morris Water Maze (3) 477-482

Saccardi, R., see Cecchi, C. (3) 651-664

Safranow, K., see Maruszak, A. (1) 197-210

Sakae, N., see Ma, L. (1) 225-237

Sánchez-Juan, P., see Vázquez-Higuera, J.L. (2) 291297

Sano, M., see Greenwood, T.A. (4) 897-907

Santos, C.O., see Verdelho, A. (3) 491-498

Sapone, A., see Puoliväli, J. (3) 499-510

Saraiva, M.J., see Oliveira, S.M. (2) 429-439

Sasiadek, M., see Zimny, A. (3) 591-601

Sattler, W., see Schweinzer, C. (2) 341-360

Sawmiller, D.R., see Chen, M. (4) 679-689

Scarpini, E., see Carecchio, M. (4) 781-790

Scarpini, E., see Cerami, C. (4) 791-797

Scazufca, M., see de Toledo Ferraz Alves, T.C. (3) 575589

Scerri, C., see Gauci, A.J. (4) 767-779

Schäuble, B., see Kavanagh, S. (3) 521-530

Scheltens, P., see Verdelho, A. (3) 491-498

Schleicher, E., see Maetzler, W. (1) 119-126

Schmeidler, J., see Greenwood, T.A. (4) 897-907

Schmidt, B., see Taghavi, A. (4) 835-843

Scholler, M., see Schweinzer, C. (2) 341-360

Schröder, J., see Seidl, U. (3) 567-574

Schulte, C., see Maetzler, W. (1) 119-126

Schweinzer, C., A. Kober, I. Lang, K. Etschmaier, M. Scholler, A. Kresse, W. Sattler and U. Panzenboeck, Processing of Endogenous AßPP in BloodBrain Barrier Endothelial Cells is Modulated by Liver-X Receptor Agonists and Altered Cellular Cholesterol Homeostasis (2) 341-360

Seeberg, G., see Reese, J.P. (4) 723-736

Seidl, U., U. Lueken, P.A. Thomann, J. Geider and J. Schröder, Autobiographical Memory Deficits in Alzheimer's Disease (3) 567-574

Selnes, P., see Grambaite, R. (2) 453-462

Serpente, M., see Carecchio, M. (4) 781-790

Serrati, C., see Picco, A. (4) 737-742

Shi, J., see Yin, J.-X. (1) 89-98

Shi, M., Y.-T. Sui, E.R. Peskind, G. Li, H. Hwang, I. Devic, C. Ginghina, J.S. Edgar, C. Pan, D.R. Goodlett, A.R. Furay, L.F. Gonzalez-Cuyar and J. Zhang, Salivary Tau Species are Potential Biomarkers of Alzheimer's Disease (2) 299305

Silverman, J.M., see Greenwood, T.A. (4) 897-907

Simonsen, A., see Mattila, J. (1) 163-176 
Sinha, M., P. Behera, P. Bhowmick, K. Banerjee, S. Basu and S. Chakrabarti, Aging Promotes Amyloid- $\beta$ Peptide Induced Mitochondrial Dysfunctions in Rat Brain: A Molecular Link Between Aging and Alzheimer's Disease (4) 753-765

Sluchanko, N.N., N.B. Gusev, Probable Participation of 14-3-3 in Tau Protein Oligomerization and Aggregation (3) 467-476

Smith, C.D., see Threlkeld, Z.D. (2) 415-427

Soejima, N., see Ma, L. (1) 225-237

Soininen, H., see Mattila, J. (1) 163-176

Soininen, H., see Yang, H. (1) 113-118

Soleti, A., see Puoliväli, J. (3) 499-510

Song, M., see Kou, J. (1) 23-38

Spisni, E., see Puoliväli, J. (3) 499-510

Squarzoni, P., see de Toledo Ferraz Alves, T.C. (3) 575589

Stapf, A.K., see Maetzler, W. (1) 119-126

Steer, C.J., see Viana, R.J.S. (1) 61-73

Stefani, M., see Cecchi, C. (3) 651-664

Sui, Y.-T., see Shi, M. (2) 299-305

Sutherland, C., see Williamson, R. (3) 615-625

Swerdlow, R.H., see Mosconi, L. (3) 483-490

Szewczyk, P., see Zimny, A. (3) 591-601

Szoeke, C., see Faux, N.G. (4) 909-922

Tabaton, M., see Guglielmotto, M. (4) 871-883

Taddei, K., see Faux, N.G. (4) 909-922

Taddei, T., see Faux, N.G. (4) 909-922

Taghavi, A., S. Nasir, M. Pickhardt, R. Heynyvon Haußen, G. Mall, E. Mandelkow, E.-M. Mandelkow and B. Schmidt, N'-BenzylideneBenzohydrazides as Novel and Selective Tau-PHF Ligands (4) 835-843

Taguchi, H., see Kou, J. (1) 23-38

Tähtivaara, L., see Puoliväli, J. (3) 499-510

Takasaki, J., K. Ono, Y. Yoshiike, M. Hirohata, T. Ikeda, A. Morinaga, A. Takashima and M. Yamada, Vitamin A has Anti-Oligomerization Effects on Amyloid- $\beta$ In Vitro (2) 271-280

Takashima, A., see Takasaki, J. (2) 271-280

Tamagno E., see Guglielmotto, M. (4) 871-883

Tamashiro-Duran, J.H., see de Toledo Ferraz Alves, T.C. (3) 575-589

Tamayev, R., see Matsuda, S. (2) 385-391

Thomann, P.A., see Seidl, U. (3) 567-574

Threlkeld, Z.D., G.A. Jicha, C.D. Smith and B.T. Gold, Task Deactivation Reductions and Atrophy within Parietal Default Mode Regions are Overlapping but Only Weakly Correlated in Mild Cognitive Impairment (2) 415-427
Tian, Q., see Wei, W. (3) 639-650

Torp, R., see Yang, J. (4) 711-722

Träber, F., see Jessen, F. (2) 393-399

Tramoni, E., see Didic, M. (1) 11-22

Tremblay, C., see Lebbadi, M. (4) 853-869

Trepanier, C.H., see Yang, K. (2) 243-252

Trounson, B.O., see Faux, N.G. (4) 909-922

Trypka., E., see Zimny, A. (3) 591-601

Turner, G.H., see Yin, J.-x. (1) 89-98

Ubhi, K., see Rockenstein, E. (4) 743-752

Usardi, A., see Williamson, R. (3) 615-625

Valdivieso, F., see Vázquez-Higuera, J.L. (2) 291-297

Valerio, D., see Greenwood, T.A. (4) 897-907

Valla, J., see Hunter, J.M. (2) 361-376

Vallada, H.P., see de Toledo Ferraz Alves, T.C. (3) 575589

van Aalten, L., see Williamson, R. (3) 615-625

Van Baelen, B., see Kavanagh, S. (3) 521-530

van der Beek, E.M., see de Wilde, M.C. (2) 327-339

van Gils, M., see Mattila, J. (1) 163-176

Vartiainen, N., see Puoliväli, J. (3) 499-510

Vassallo, N., see Gauci, A.J. (4) 767-779

Vázquez-Higuera, J.L., I. Mateo, P. Sánchez-Juan, E. Rodríguez-Rodríguez, A. Pozueta, M. Calero, J.L. Dobato, A. Frank-García, F. Valdivieso, J. Berciano, M.J. Bullido and O. Combarros, Genetic Variation in the Tau Kinases Pathway May Modify the Risk and Age at Onset of Alzheimer's Disease (2) 291-297

Verbeek, M.M., see Aerts, M.B. (2) 377-384

Verdelho, A., S. Madureira, C. Moleiro, C.O. Santos, J.M. Ferro, T. Erkinjuntti, A. Poggesi, L. Pantoni, F. Fazekas, P. Scheltens, G. Waldemar, A. Wallin, and D. Inzitari, Self-Perceived Memory Complaints Predict Progression to Alzheimer Disease. The LADIS Study (3) 491-498

Viana, R.J.S., C.J. Steer and C.M.P. Rodrigues, AmyloidB Peptide-Induced Secretion of Endoplasmic Reticulum Chaperone Glycoprotein GRP94 (1) 61-73

Vilaseca, M.A., see Ortez, C. (2)253-257

Villa, C., see Cerami, C. (4) 791-797

Villar, C., see Ortez, C. (2) 253-257

Villemagne, V.L., see Darby, D.G. (3) 627-637

Villemagne, V.L., see Faux, N.G. (4) 909-922

Viña, J., see Lloret, A. (4) 701-709

Virkki, A., see Mattila, J. (1) 163-176

Vizcaychipi, M.P., see Pac-Soo, C. (1) 1-10 
Wajngarten, M., see de Toledo Ferraz Alves, T.C. (3) 575-589

Waldemar, G., see Mattila, J. (1) 163-176

Waldemar, G., see Verdelho, A. (3) 491-498

Wallin, A., see Bjerke, M. (3) 665-676

Wallin, A., see Verdelho, A. (3) 491-498

Wan, C.-M., see Guan, J.-W. (4) 799-807

Wang, J., see Li, C. (2) 281-290

Wang, J.-Z., see Wei, W. (3) 639-650

Wang, L., see Du, X.-t. (2) 401-413

Wang, Q., see Wei, W. (3) 639-650

Wang, Y.-j., see Du, X.-t. (2) 401-413

Wang, Z.-R., see Guan, J.-W. (4) 799-807

Ward, V., see Faux, N.G. (4) 909-922

Wei, W., Y.-H. Liu, C.-E. Zhang, Q. Wang, Z. Wei, D.D. Mousseau, J.-Z. Wang, Q. Tian and G.-P. Liu, Folate/vitamin-B12 Prevents Chronic Hyperhomocysteinemia-Induced Tau Hyperphosphorylation and Memory Deficits in Aged Rats (3) 639-650

Wei, Z., see Wei, W. (3) 639-650

Weinberg, D.D., see Mielke, M.M. (2) 259-269

Wille, E., see Dumont, M. (1) 211-223

Williamson, R., L. van Aalten, D.M.A. Mann, B. Platt, F. Plattner, L. Bedford, J. Mayer, D. Howlett, A. Usardi, C. Sutherland and A.R. Cole, CRMP2 Hyperphosphorylation is Characteristic of Alzheimer's Disease and not a Feature Common to Other Neurodegenerative Diseases (3) 615-625

Wiltfang, J., see Jessen, F. (2) 393-399

Wojtynska, R., see Zimny, A. (3) 591-601

Woodward, M., see Darby, D.G. (3) 627-637

Wright, D., see Cecchi, C. (3) 651-664

Wu, J., see Bihaqi, S.W. (4) 819-833

Wurster, I., see Maetzler, W. (1) 119-126

Xi Chen, J., see Alikhani, N. (1) 75-87

Xu, Y., see Yang, J. (4) 711-722

Xue, D., see Du, X.-t. (2) 401-413

Yamada, M., see Takasaki, J. (2) 271-280

Yamasaki, R., see Ma, L. (1) 225-237

Yan, S., see Alikhani, N. (1) 75-87

Yan, S.S., see Alikhani, N. (1) 75-87

Yanagihara, Y.T., see Ma, L. (1) 225-237

Yang, J., L.K. Lunde, P. Nuntagij, T. Oguchi, L.M.A. Camassa, L.N.G. Nilsson, L. Lannfelt, Y. Xu, M.
Amiry-Moghaddam, O.P. Ottersen and R. Torp, Loss of Astrocyte Polarization in the Tg-ArcSwe Mouse Model of Alzheimer's Disease (4) 711722

Yang, K., J. Belrose, C.H. Trepanier, G. Lei, M.F. Jackson and J.F. MacDonald, Fyn, a Potential Target for Alzheimer's Disease (2) 243-252

Yang, Y., see Du, X.-t. (2) 401-413

Yang, H., Y. Lyutvinskiy, H. Soininen and R.A. Zubarev, Alzheimer's Disease and Mild Cognitive Impairment are Associated with Elevated Levels of Isoaspartyl Residues in Blood Plasma Proteins (1) $113-118$

Yesavage, J.A., see David, R. (1) 155-161

Yin, J.-X., G.H. Turner, H.-j. Lin, S.W. Coons and J. Shi, Deficits in Spatial Learning and Memory is Associated with Hippocampal Volume Loss in Aged Apolipoprotein E4 Mice (1) 89-98

Yoshiike, Y., see Takasaki, J. (2) 271-280

You, C., see Guan, J.-W. (4) 799-807

Youssef, A.M., see Hashioka, S. (3) 531-541

Yrjänheikki, J., see Puoliväli, J. (3) 499-510

Yu, F., see Dumont, M. (1) 211-223

Yuen, W.B., see Saab, B.J. (3) 477-482

Yun, Y.P., see Choi, I.S. (1) 127-141

Zaidi, N., see Mielke, M.M. (2) 259-269

Zampagni, M., see Cecchi, C. (3) 651-664

Zawia, N.H., see Bihaqi, S.W. (4) 819-833

Zeitzer, J.M., see David, R. (1) 155-161

Żekanowski, C., see Maruszak, A. (1) 197-210

Zetterberg, H., see Bjerke, M. (3) 665-676

Zhan, D.-w., see Du, X.-t. (2) 401-413

Zhang, C.-E., see Wei, W. (3) 639-650

Zhang, J., see Shi, M. (2) 299-305

Zhao, L., see Gao, B. (2) 317-326

Zhao, M., see Du, X.-t. (2) 401-413

Zheng, J., see Li, C. (2) 281-290

Zimny, A., P. Szewczyk, E. Trypka., R. Wojtynska, L. Noga, J. Leszek and M. Sasiadek, Multimodal Imaging in Diagnosis of Alzheimer's Disease and Amnestic Mild Cognitive Impairment: Value of Magnetic Resonance Spectroscopy, Perfusion, and Diffusion Tensor Imaging of the Posterior Cingulate Region (3) 591-601

Zubarev, R.A., see Yang, H. (1) 113-118 